\title{
Prevalência das infecções sexualmente transmissíveis na população idosa da Amazônia Brasileira
}

\author{
Prevalence of sexually transmitted infections in the aged population of Brazilian Amazon \\ Prevalencia de infecciones de transmisión sexual en la población mayor de la Amazonía \\ Brasileña
}

Manuelle Paes Mebius ${ }^{1 *}$, Luis Enrique Bermejo Galan ${ }^{1}$, Bianca Jorge Sequeira Costa ${ }^{1}$, Lilian Mara Vieira Monsalve Moraga ${ }^{1}$.

\section{RESUMO}

Objetivo: Estimar a prevalência e os possíveis fatores de risco associados às Infecções Sexualmente Transmissíveis (IST) na terceira idade. Métodos: Trata-se de estudo transversal, analítico de caráter quantitativo, realizado na região norte do Brasil, no período de março a dezembro de 2019, com 200 idosos, os quais foram submetidos a questionários e realização de testes rápidos para Sífilis, Hepatite $B$, Hepatite $C$ e Vírus da Imunodeficiência Humana (HIV). Resultados: A prevalência das Infecções Sexualmente Transmissíveis (IST) foi de $6 \%$ e entre as 12 ocorrências, foram observados 9 casos $(4,5 \%)$ de sífilis, dois (1\%) de hepatite C e um $(0,5 \%)$ de infecção pelo HIV. Houve associação entre ter IST e ser homem, ter relacionamento fixo e não usar preservativo rotineiramente. Conclusão: Os resultados demonstraram um elevado percentual de ISTs entre idosos associadas a vários fatores, evidenciando situações de vulnerabilidade dessa população. Propõe-se educação contínua por meio dos profissionais de saúde, abordando vários aspectos da sexualidade, formas de prevenção e transmissão das IST, além do diagnóstico precoce, proporcionando assim uma melhor qualidade de vida a essa população.

Palavras-chave: Idosos, Infecção sexualmente transmissível, Infecção.

\begin{abstract}
Objective: To estimate the prevalence and possible risk factors associated with Sexually Transmitted Infections (STIs) in old age. Methods: This is a cross-sectional, analytical study of a quantitative character, carried out in the northern region of Brazil, from March to December 2019, with 200 elderly people, who were submitted to questionnaires and rapid tests for Syphilis, Hepatitis B, Hepatitis $\mathrm{C}$ and Human Immunodeficiency Virus (HIV). Results: The prevalence of Sexually Transmitted Infections (STI) was 6\% and among the 12 occurrences, 9 cases $(4.5 \%)$ of syphilis were observed, two (1\%) of hepatitis $\mathrm{C}$ and one $(0.5 \%) \mathrm{HIV}$ infection. There was an association between having STIs and being a man, having a fixed relationship and not using condoms routinely. Conclusion: The results showed a high percentage of STIs among the elderly associated with several factors, showing situations of vulnerability in this population. Continuous education is proposed through health professionals, addressing various aspects of sexuality, ways of preventing and transmitting STIs, in addition to early diagnosis, thus providing a better quality of life for this population.
\end{abstract}

Key words: Aged, Sexually transmitted infection, Infection.

\section{RESUMEN}

Objetivo: Estimar la prevalencia y los posibles factores de riesgo asociados a las Infecciones de Transmisión Sexual (ITS) en la vejez. Métodos: Se trata de un estudio transversal, analítico de carácter cuantitativo,

1Universidade Federal de Roraima (UFRR), Boa Vista - RR. *E-mail: bianca.costa@ufrr.br 
realizado en la región norte de Brasil, de marzo a diciembre de 2019, con 200 ancianos, quienes fueron sometidos a cuestionarios y pruebas rápidas para Sífilis, Hepatitis B, Virus de la hepatitis $\mathrm{C}$ y de la inmunodeficiencia humana (VIH). Resultados: La prevalencia de Infecciones de Transmisión Sexual (ITS) fue de $6 \%$ y entre las 12 ocurrencias se observaron 9 casos (4.5\%) de sífilis, dos (1\%) de hepatitis $C$ y una $(0.5 \%)$ de infección por VIH. Hubo una asociación entre tener ITS y ser hombre, tener una relación fija y no usar condones de manera rutinaria. Conclusión: Los resultados mostraron un alto porcentaje de ITS entre los adultos mayores asociados a varios factores, mostrando situaciones de vulnerabilidad en esta población. Se propone la educación continua a través de los profesionales de la salud, abordando diversos aspectos de la sexualidad, formas de prevenir y transmitir las ITS, además del diagnóstico precoz, brindando así una mejor calidad de vida a esta población.

Palabras clave: Anciano, Infección de transmisión sexual, Infección.

\section{INTRODUÇÃO}

A população brasileira sofreu intensas modificações a partir da metade do século XX. Entre as décadas de 1950 e 1960 a combinação da redução da mortalidade com a manutenção dos elevados níveis de fecundidade e natalidade proporcionaram ao país uma das mais elevadas taxas de crescimento populacional da história. Após esse período, entre os anos de 1980 e 2010, devido à queda nas taxas de mortalidade e fecundidade, houve uma mudança deste perfil, que conduziu ao envelhecimento populacional, com predomínio cada vez maior de pessoas acima de 60 anos (VASCONCELOS AM e GOMES MM, 2012).

De acordo com Suzman R, et al. (2015), com o passar dos anos, o envelhecimento das populações se tornará o próximo desafio de saúde pública no mundo e pela primeira vez na história, o número de pessoas com idade acima de 65 anos será superior ao de crianças menores de 5 anos. Tal fato está relacionado aos avanços na medicina e ao desenvolvimento socioeconômico, que diminuíram consideravelmente as taxas de morbimortalidade de origem infecciosa. Essas transformações demográficas e epidemiológicas, aliadas ao acelerado processo de urbanização, modernização, globalização e mudanças concomitantes nos fatores de risco e estilos de vida, aumentaram a vulnerabilidade das condições de saúde dessa população.

O aumento da população idosa traz consigo uma série de desafios aos sistemas de saúde pública, uma vez que o envelhecimento não é sinônimo de adoecimento, a não ser que haja doença associada. Nas últimas décadas, a velhice tem sido relacionada com boa qualidade de vida, possivelmente devido aos avanços tecnológicos e na área da saúde, que permitem o acesso aos serviços públicos ou privados (MIRANDA GM, et al., 2016).

A abordagem da sexualidade como algo natural entre idosos ainda é rodeada de preconceitos, produzindo um estereótipo errado de que o prazer sexual esteja limitado somente ao período juvenil da vida (TERRA NL, 2014). Ao discutir sobre a sexualidade dos idosos é importante lembrar que a maioria deles herdou de outras gerações informações diferenciadas sobre esse assunto, as quais designavam a sexualidade como algo exclusivamente relacionado à procriação, além de aspectos religiosos que também sustentaram essa visão limitada do sexo, associando as experiências sexuais a algo pecaminoso (VIEIRA KFL, et al., 2016).

É incorreto que a velhice subtraia o interesse pelas relações sexuais e que sua expressão física deva terminar, sendo assim de fundamental importância para o idoso manter uma vida sexual ativa até o fim da vida. Muitas vezes, o prazer sexual poder ser considerado um dos melhores motivos para continuar vivendo e dar sentido aos últimos anos de sua existência (PASCUAL CP, 2002).

Dentre as várias conquistas que essa faixa etária vem alcançando nos últimos anos, o seguimento da vida sexual e a melhoria na qualidade de vida são muito importantes, aliados ao progresso relacionado aos serviços de saúde, tais como terapia de reposição hormonal para mulheres na pós-menopausa e o acesso às medicações para disfunção erétil, tornando possível a continuidade do sexo entre idosos (LAROQUE MF, et al., 2011). 
A insuficiência de dados epidemiológicos e programas voltados à prevenção agregados à continuidade da vida sexual ativa, questões comportamentais e a própria dinâmica fisiológica do envelhecimento, têm contribuído para o aumento das infecções sexualmente transmissíveis (ISTs) na terceira idade. Existem poucos dados literários sobre IST em idosos, além disso grande parte dos trabalhos publicados concentra informações apenas sobre HIV/Aids, esquecendo das outras infecções e a maioria dessas referências são oriundas de pesquisas com populações específicas realizadas em ambulatórios, constatando assim um déficit de estudos multicêntricos. Diversos estudos acerca deste tema denotam um aumento das IST na terceira idade em vários países (DORNELAS JN, et al., 2015).

Brito NM, et al. (2016) apresentaram em sua pesquisa que grande parcela dos indivíduos com mais de 60 anos de idade possui pouca instrução sobre as formas de transmissão e prevenção das infecções sexualmente transmissíveis, fato este que pode colaborar para o aumento da suscetibilidade e do risco de contaminação, acreditando serem pessoas de baixo risco para adquirir este tipo de infecções.

É de extrema necessidade que os profissionais da área da saúde conheçam os problemas relacionados à sexualidade dos idosos para poder orientá-los. Apesar dos novos conhecimentos acerca da manutenção, da concepção e da prática sexual nesta faixa etária, a maioria dos profissionais, não têm como prática incluir questionamentos sobre o tema, corroborando o preconceito da velhice assexuada, deixando de abordar assuntos sobre a libido e a sexualidade em geral com essa população. Em virtude disso, muitos idosos sentem-se constrangidos em expor suas dúvidas, e a falta de comunicação entre esses dois elos mostra a incapacidade em abordar esta temática por todos de forma esclarecedora e respeitosa (TERRA NL, 2014).

Desta forma, com base no cenário exposto, o presente estudo visa estimar a prevalência e os possíveis fatores de risco associados às Infecções Sexualmente Transmissíveis (ISTs) na terceira idade em uma capital da Amazônia brasileira.

\section{MÉTODOS}

Trata-se de um estudo transversal, prospectivo, analítico, de caráter quantitativo que objetivou avaliar a ocorrência de ISTs (Sífilis, Hepatites B e C e HIV/Aids) na terceira idade. A pesquisa foi realizada em uma capital da Amazônia brasileira.

O grupo amostral foi constituído por 200 pacientes com idade superior a 60 anos, cadastrados em três Unidades Básicas de Saúde (UBS), além de uma clínica especializada, pública. Para definir o quantitativo amostral levou-se em consideração o número de pacientes idosos cadastrados nessas unidades de saúde especificadas.

Os participantes deste estudo foram abordados por meio de convite verbal enquanto aguardavam atendimento médico nas instituições de saúde. Os critérios de inclusão foram: ter praticado relação sexual em algum momento da vida e possuir faculdades cognitivas preservadas; foram excluídos pacientes indígenas, estrangeiros, portadores de transtornos neurocognitivo que pudessem dificultar o entendimento dos objetivos do estudo e a qualidade dos dados a serem obtidos e aqueles que não assinaram o Termo de Consentimento Livre e Esclarecido (TCLE).

Os dados foram obtidos no período de abril a dezembro de 2019, através de entrevista individual, sempre em local privativo. O recurso utilizado para a coleta de dados foi o questionário utilizado previamente por Andrade J, et al. (2017) e readaptado a esta pesquisa. Somente após a assinatura do TCLE, os participantes foram convidados a responder o questionário e posteriormente submetidos a realização dos testes rápidos.

Os testes rápidos, realizados por punção digital, foram: teste rápido treponêmico para sífilis (sífilis), HBsAg (hepatite B), Anti-HCV (hepatite C) e Anti-HIV 1 e 2 (HIV/AIDS). Vale ressaltar que todos os testes foram realizados seguindo as especificações preconizadas pelos fabricantes.

Os pacientes com resultados reagentes nos testes realizados foram encaminhados ao Serviço de Assistência Especializada (SAE), referência estadual para o acompanhamento desses casos. Ressalta-se que para considerar reagente o Anti-HIV 1 e 2, foi necessário realizar um segundo teste rápido; nos casos de 
sífilis, foi solicitado exame complementar de Venereal Disease Research Laboratory (VDLR); para as demais ISTs estudadas somente foi realizado um teste rápido. Tendo em vista as questões éticas envolvidas nesta pesquisa, todos os participantes deste estudo receberam aconselhamento pré-teste, sendo inteirados do caráter sigiloso da testagem e orientados sobre janela imunológica e outros aspectos das IST a serem testadas, além das possibilidades de resultados. Após a realização dos testes, para os casos identificados como reagentes, disponibilizou-se suporte psicológico, esclarecimento de dúvidas e outras orientações individualizadas para cada caso.

A presença de IST foi a variável de desfecho da pesquisa. Para a análise descritiva dos dados foram consideradas a caracterização sociodemográfica e a conduta sexual dos idosos: gênero, idade, raça, escolaridade, renda mensal, possuir parceiro fixo, ser sexualmente ativo, história pregressa de IST, relacionamento extraconjugal, uso de preservativo e realização de testes rápidos ou sorologias prévias para IST. A análise estatística inferencial foi realizada a partir dos testes qui-quadrado ou exato de Fisher, realizada pelo programa BioEstat 5.0.

Este estudo foi aprovado pelo Comitê de Ética em Pesquisa, da Universidade Federal de Roraima, sob o Parecer no 3.206.031.

\section{RESULTADOS}

A prevalência das ISTs na população estudada foi de $6 \%$, ou seja, 12 casos reagentes, correspondendo a 1 caso de HIV, 2 casos de Hepatite $C$ e 9 casos de sífilis. Não foi evidenciado nenhum caso de Hepatite B. Tal distribuição pode ser visualizada por meio do Gráfico 1.

Gráfico 1 - Prevalência das ISTs entre os participantes do estudo.

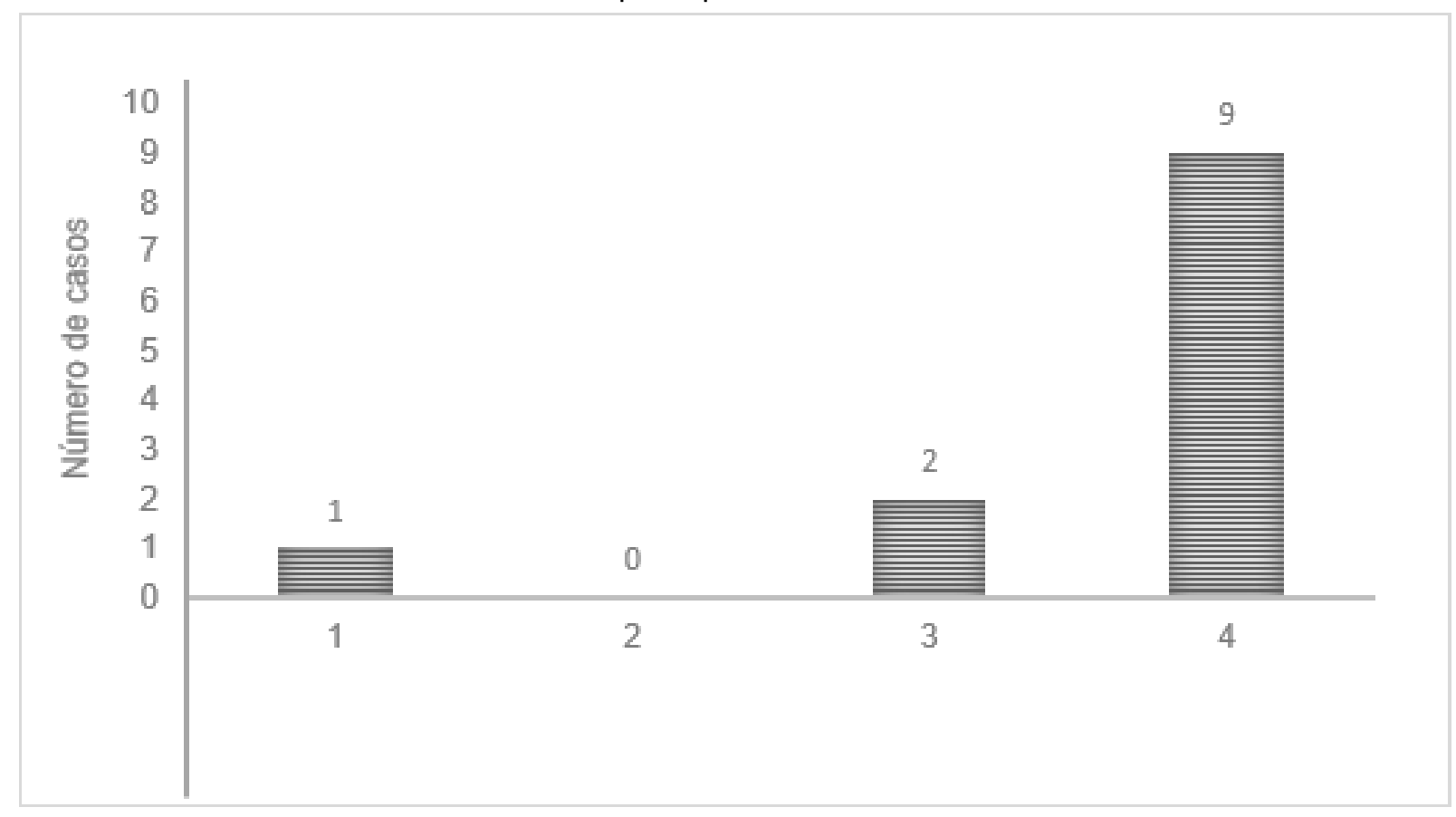

Fonte: Mebius MP, et al., 2021

A seguir demonstra-se a análise univariada relacionando à prevalência das ISTs com as características sociodemográficas e comportamentais dos idosos. Observa-se que dentre todas as variáveis avaliadas, somente o sexo, ter um parceiro sexual fixo e o uso de preservativo demonstraram associação com o desfecho (Tabela 1). 
Tabela 1 - Análise univariada das características sociodemográficas e comportamentais dos idosos associadas à ocorrência de ISTs.

\begin{tabular}{|c|c|c|c|c|c|}
\hline \multirow{3}{*}{ Variáveis } & \multicolumn{4}{|c|}{ IST } & \multirow{3}{*}{$p$-value } \\
\hline & \multicolumn{2}{|c|}{ Sim } & \multicolumn{2}{|c|}{ Não } & \\
\hline & $\mathbf{N}$ & $\%$ & $\mathbf{N}$ & $\%$ & \\
\hline \multicolumn{6}{|l|}{ Sexo } \\
\hline Masculino & 10 & $5,0 \%$ & 91 & $45,5 \%$ & \multirow{2}{*}{0,04} \\
\hline Feminino & 2 & $1,0 \%$ & 97 & $48,5 \%$ & \\
\hline \multicolumn{6}{|l|}{ Idade (anos) } \\
\hline 60 a 74 & 10 & $5,0 \%$ & 160 & $80,0 \%$ & \multirow{2}{*}{0,80} \\
\hline$>=75$ & 2 & $1,0 \%$ & 28 & $14,0 \%$ & \\
\hline \multicolumn{6}{|l|}{ Raça (cor) } \\
\hline Branca & 2 & $1,0 \%$ & 59 & $29,5 \%$ & \multirow{2}{*}{0,45} \\
\hline Outra (preta, parda, amarela) & 10 & $5,0 \%$ & 129 & $64,5 \%$ & \\
\hline \multicolumn{6}{|l|}{ Escolaridade (anos) } \\
\hline 0 a 3 & 1 & $0,5 \%$ & 61 & $30,5 \%$ & \multirow{3}{*}{0,13} \\
\hline 4 a 7 & 8 & $4,0 \%$ & 76 & $38,0 \%$ & \\
\hline 8 ou mais & 3 & $1,5 \%$ & 51 & $25,5 \%$ & \\
\hline \multicolumn{6}{|l|}{ Renda >2 salários mínimos } \\
\hline Sim & 6 & $3,4 \%$ & 59 & $33,7 \%$ & \multirow{2}{*}{0,51} \\
\hline Não & 6 & $3,4 \%$ & 104 & $59,4 \%$ & \\
\hline \multicolumn{6}{|l|}{ Relação sexual } \\
\hline Sim & 11 & $5,5 \%$ & 138 & $69,0 \%$ & \multirow{2}{*}{0,28} \\
\hline Não & 1 & $0,5 \%$ & 50 & $25,0 \%$ & \\
\hline \multicolumn{6}{|l|}{ Parceiro Fixo } \\
\hline Sim & 7 & $3,5 \%$ & 114 & $57,0 \%$ & \multirow{2}{*}{0,02} \\
\hline Não & 5 & $2,5 \%$ & 74 & $37,0 \%$ & \\
\hline \multicolumn{6}{|c|}{ Estado Cívil e/ou União estável } \\
\hline Casado (a) & 5 & $2,5 \%$ & 108 & $54,0 \%$ & \multirow{2}{*}{0,44} \\
\hline Outros & 7 & $3,5 \%$ & 80 & $40,0 \%$ & \\
\hline \multicolumn{6}{|l|}{ História de IST } \\
\hline Sim & 3 & $1,5 \%$ & 34 & $17,0 \%$ & \multirow{2}{*}{0,54} \\
\hline Não & 9 & $4,5 \%$ & 154 & $77,0 \%$ & \\
\hline \multicolumn{6}{|l|}{ Uso de Preservativo } \\
\hline Usou sempre & 0 & $0,0 \%$ & 3 & $2 \%$ & \multirow{3}{*}{0,02} \\
\hline Às vezes & 8 & $4,0 \%$ & 55 & $28 \%$ & \\
\hline Nunca usou & 4 & $2,0 \%$ & 130 & $65 \%$ & \\
\hline \multicolumn{6}{|l|}{ TR $^{\star}$ ou sorologia prévia } \\
\hline Sim & 5 & $2,5 \%$ & 41 & $20,5 \%$ & \multirow{2}{*}{0,11} \\
\hline Não & 7 & $3,5 \%$ & 147 & $73,5 \%$ & \\
\hline
\end{tabular}

Fonte: Mebius MP, et al., 2021.

Com relação ao gênero dos 200 participantes do estudo, a distribuição foi semelhante, uma vez que 50,5\% dos participantes são do sexo masculino e $49,5 \%$ do sexo feminino. A idade dos participantes variou entre 60 e 82 anos, sendo a faixa etária de 60 a 74 anos a mais prevalente (85,0\%). A maior parte dos entrevistados $(69,5 \%)$ não se considerou de raça branca, uma vez que se autodeclararam como sendo pretos pardos ou amarelos.

Em relação ao nível de escolaridade, 31,0\% informou que tinha até 3 anos de estudo, $42,0 \%$ referiu ter cursado entre 4 e 7 anos e $27,0 \%$ declarou ter estudado por 8 ou mais anos. No que diz respeito à remuneração, $87,5 \%$ possuíam renda, destes, $55,0 \%$ recebia entre 1 a 2 salários mínimos e $32,5 \%$ recebia mais de 2 salários mínimos. No que tange à atividade sexual, $74,5 \%$ dos participantes referiu ser sexualmente ativo, destes, 60,5\% relatou ter parceiro fixo, 56,5\% tinha relacionamento estável e 16,5\% relatou manter relações extraconjugais. Quanto à história de IST, 18,5\% referiu já ter adquirido alguma ao longo da vida, entre elas gonorreia, Papiloma Vírus Humano (HPV), herpes e sífilis. Chama a atenção que $77 \%$ referiu nunca ter realizado testes para diagnosticar IST. 
As variáveis sociodemográficas e comportamentais possivelmente associadas ao resultado reagente para IST foram: ser do sexo masculino, ter um parceiro sexual fixo e não usar preservativo rotineiramente. Conforme visualizado no Gráfico 2, o não uso do preservativo foi associado aos seguintes motivos:

Gráfico 2 - Motivos informados pelos participantes do estudo para o não uso do preservativo.

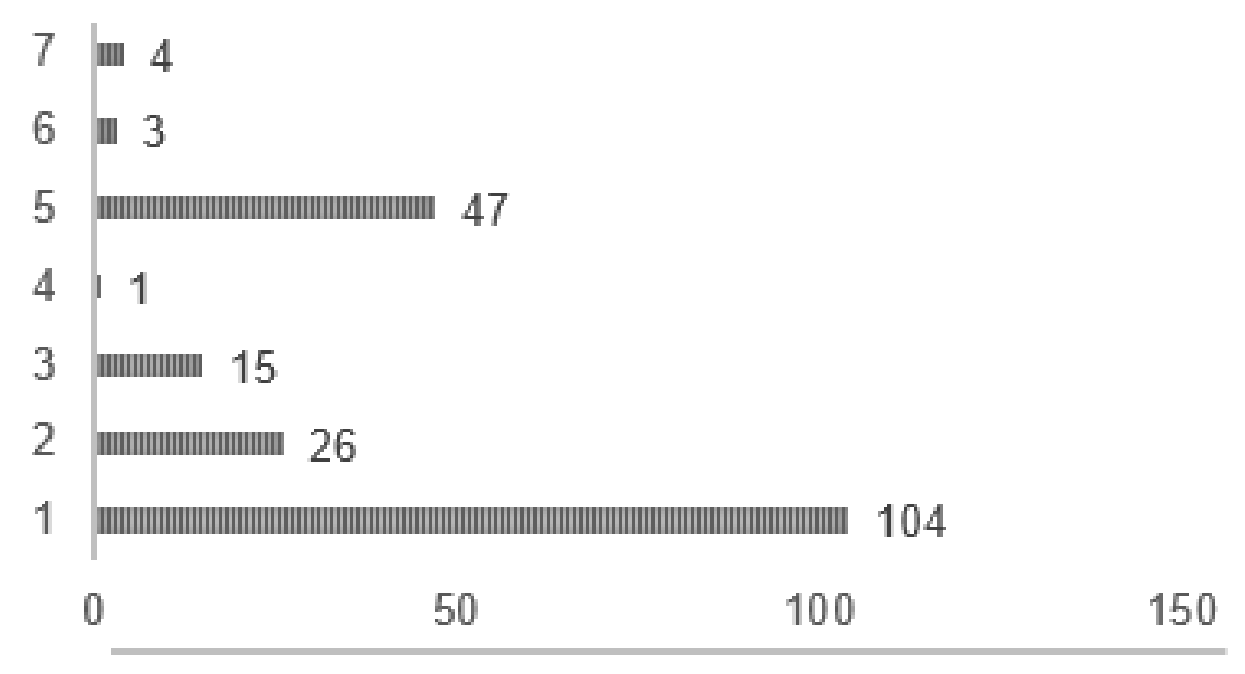

Fonte: Mebius MP, et al., 2021.

Possuir parceiro fixo também parece estar relacionado a maiores chances de adquirir IST. Outra associação significativa observada foi o predomínio das IST no gênero masculino, sendo 3 vezes mais comum que no feminino. A relação das IST por sexo e faixa etária constam no Gráfico 3.

Gráfico 3 - IST segundo gênero e faixa etária dos participantes do estudo.

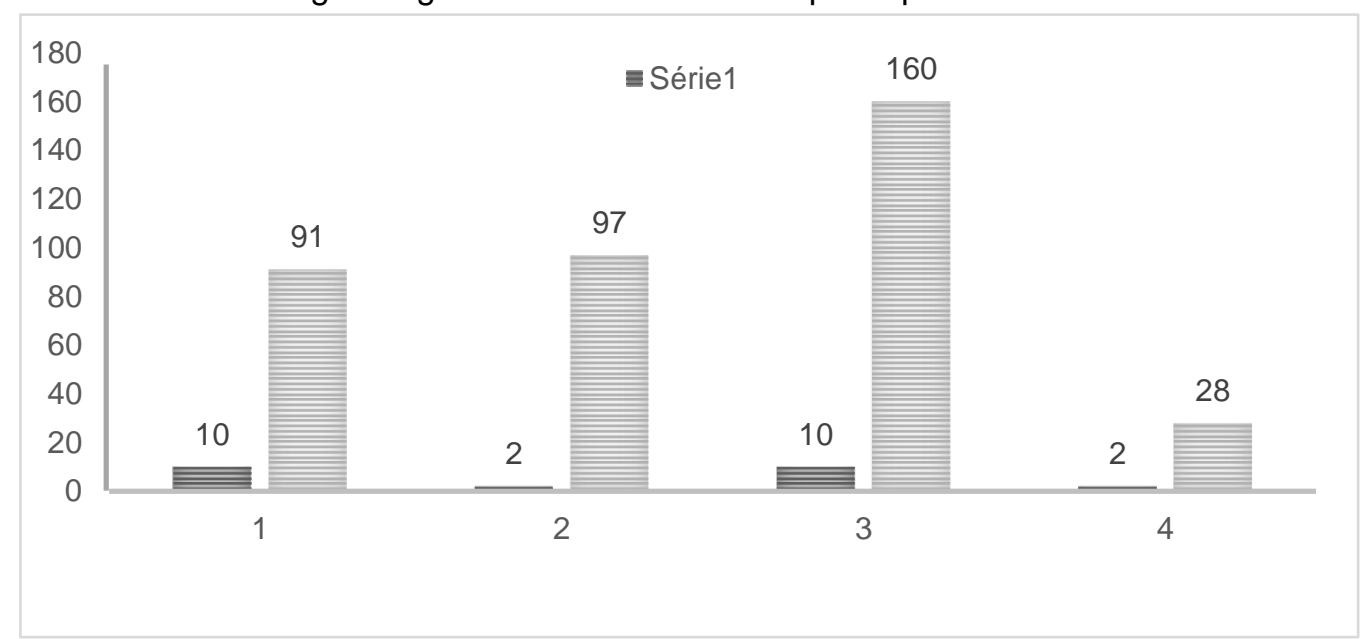

Fonte: Mebius MP, et al., 2021.

\section{DISCUSSÃO}

Este estudo identificou a prevalência de $6 \%$ para ocorrência de ISTs entre pessoas com idade superior a 60 anos, sendo maior que a demonstrada em um estudo realizado no interior paulista $(3,4 \%)$ e inferior à verificada em um outro estudo em um município de médio porte da Bahia (25,32\%), respectivamente realizados por (ANDRADE J, et al., 2017; FERREIRA CD, et al., 2019). 
Semelhante ao estudo do Andrade J, et al. (2017), evidenciou-se um número maior de casos de sífilis em relação às outras IST, diferindo do estudo realizado na Bahia que observou maior frequência das hepatites virais B e C (FERREIRA CD, et al., 2019). Estes dados são corroborados por dados epidemiológicos nacionais, que indicam que a capital onde foi realizado o presente estudo é a sexta com maior taxa de detecção de sífilis adquirida no Brasil (BRASIL, 2019a).

É notório mencionar que o boletim epidemiológico não traz uma informação precisa acerca da faixa etária acima de 60 anos, no entanto, destaca uma taxa de detecção ascendente a partir dos 50 anos de idade (BRASIL, 2019). Um estudo realizado em Sobral-CE por Bastos LM, et al. (2018) evidenciou que apesar da sífilis ser uma doença com uma prevalência maior na população adulta e jovem, uma grande proporção dos participantes, incluindo idosos, afirmou não conhecer a doença e não saber sua forma de transmissão.

Embora o HIV represente um percentual baixo neste estudo, é de grande importância sua identificação, uma vez que os dados demonstram uma tendência de crescimento linear deste agravo na população idosa nos últimos anos (SILVA BN, et al., 2018). Segundo dados do boletim epidemiológico de HIV/AIDS, em 2009 havia 569 casos em idosos no Brasil e atualmente há 9.397 casos nessa faixa etária (BRASIL, 2019b). Em estudo de Affeldt $A B$, et al. (2015), realizado em Pelotas-RS, verificou-se que a maior parte dos pacientes idosos diagnosticados com HIV já se apresentava com a imunodeficiência avançada, ou seja, mais que números é uma situação de saúde pública que requer atenção pela grande vulnerabilidade na qual se encontram os idosos portadores do HIV, que pode levá-los a desenvolver formas graves da doença.

Cerqueira MR, et al. (2016) identificaram que a principal forma de diagnóstico do HIV no idoso se dá após a descoberta do status de soropositivo do parceiro e às vezes após a morte em decorrência da AIDS. Outras formas de diagnóstico são durante a investigação de doenças oportunistas como a tuberculose e quando realizados exames de rotina que contemplem sorologia anti-HIV. Estes fatos demonstram que ainda se faz necessário incentivar o diagnóstico precoce não somente do HIV como também das outras ISTs, como forma de promover uma melhor qualidade de vida para os idosos.

Diante de dois casos de hepatite $\mathrm{C}$ identificados na pesquisa, é importante frisar que a capital do estado de Roraima encontra-se entre as nove capitais brasileiras com taxas de detecção maior que a média nacional para esta infecção. No país, no período de 2008 a 2018, observou-se que o número de casos notificados ocorreu principalmente a partir dos 60 anos. Quanto aos mecanismos de transmissão, em 2018 houve uma mudança em relação aos anos anteriores, uma vez que a proporção de infecções por relação sexual foi superior aos percentuais relacionados com uso de drogas e transfusões (BRASIL, 2019c).

Durante o estudo não foram identificados casos de hepatite B entre os idosos; como hipótese para tal pode-se inferir que a cobertura vacinal, no município de realização da pesquisa, esteja abrangendo de forma eficiente esse grupo populacional, o que os torna menos susceptíveis a essa infecção. $O$ boletim epidemiológico mais recente constatou que o Brasil vem apresentando nos últimos anos pouca variabilidade nas taxas de detecção para hepatite B, inclusive com tendência de queda a partir de 2014 (BRASIL, 2019c).

O não uso do preservativo foi considerado uma das principais variáveis que pode estar relacionada ao risco de adquirir uma IST. Constatou-se que $65 \%$ dos idosos nunca fez uso do preservativo e que $28 \%$ só o utilizou esporadicamente. A principal justificativa mencionada pelos participantes para este fato foi a confiança no parceiro, seguida da disfunção sexual. Esses resultados vão ao encontro de outros estudos, nos quais mais $70 \%$ dos idosos revelou não fazer uso de preservativo como método de proteção (GNOATTO FR, et al., 2020, CERQUEIRA MR, et al., 2016).

Apesar dos idosos conhecerem os riscos aos quais estão expostos, nem sempre fazem uso do preservativo, acreditando ser desnecessário por terem relacionamento estável. Ademais, existe ainda forte resistência ao uso deste mecanismo de proteção, visto que alimentam a falsa impressão da inutilidade deste na relação sexual, uma vez que mulheres com idade mais avançada não podem mais engravidar (THEIS LC e GOUVÊA DL, 2019). A idade avançada demonstra ainda que essa parcela da população possui dificuldades na utilização do método de barreira, associando também à diminuição da sensibilidade e perda da ereção (AGUIAR RB et al., 2020). Percebe-se que não existe um fator isolado que justifique a não adesão ao uso do 
preservativo, existem sim, diversos fatores pessoais e culturais que vem contribuindo para o não uso e consequentemente um aumento nas prevalências das infecções sexuais na terceira idade.

Este estudo demonstrou que o número de casos de IST foi maior em pessoas que tinham parceiro fixo. Os idosos consideram a ideia do relacionamento fixo como uma forma de prevenção das IST, julgando desnecessário o uso da camisinha; além disso, acreditam que é um modo de demonstrar confiança e ao mesmo tempo fidelidade ao cônjuge, o que torna a relação desprotegida, por medo, vergonha ou por gerar desconfiança de infidelidade (MOURA MMS et al., 2014).

Com relação à caracterização do perfil sociodemográfico, embora a distribuição entre os gêneros dos participantes da pesquisa tenha sido semelhante, com 50,5\% do sexo masculino e $49,5 \%$ do feminino, constatou-se um predomínio maior de IST nos homens, com chances três vezes maior de adquirir uma infecção sexual do que as mulheres, em consonância com resultados de Ferreira CD, et al. (2019) e Alencar RA (2012). Entretanto, distingue-se dos estudos de Andrade J, et al. (2017), no qual o predomínio foi maior no sexo feminino. O resultado do presente estudo pode ter relação com uma maior frequência sexual dentre os homens quando comparado ao sexo feminino, fato que pode estar associado ao uso de medicamentos para disfunção erétil, favorecendo continuidade das relações sexuais (MOURA DS, et al., 2017).

Mesmo os idosos tendo um relacionamento estável, sendo casados e possuindo um parceiro fixo, foi bastante comum, neste estudo, o relato da ocorrência de relações extraconjugais. Segundo Araújo CAD (2018), uma alternativa encontrada pelos idosos do sexo masculino para manter-se em um relacionamento duradouro é a infidelidade, uma vez que muitos deles relatam a diminuição do interesse sexual por parte de suas companheiras. Vale ressaltar que a ocorrência desses casos extraconjugais não resulta somente da insatisfação no matrimônio, advém de uma cultura complacente na qual foram educados, que permite ao homem uma maior permissibilidade sexual e como resultado aliado a não utilização do preservativo tem-se uma maior vulnerabilidade destes e principalmente de suas companheiras para IST.

Quanto à faixa etária dos participantes, verificou-se que a maioria possuía idade inferior a 75 anos, semelhante a outros estudos (FERREIRA CO, et al., 2019, SILVA JDB, et al., 2017, Andrade J, et al., 2017). Em relação a raça, esta pesquisa aponta um predomínio de pretos, pardos e amarelos corroborando com Ferreira CO, et al. (2019) e Silva MP (2014). Essa predominância de raças não brancas possivelmente está relacionada ao perfil populacional da região norte, onde existe uma grande miscigenação, o que contrasta outros estudos onde houve predomínio de brancos (ANDRADE J, et al., 2017).

A escolaridade dos idosos foi representada por diferentes níveis, com discreto predomínio daqueles que cursaram apenas o ensino básico e fundamental. Entretanto, não foi evidenciada associação entre o nível de escolaridade e o diagnóstico reagente para ISTs, fato que diverge de outros estudos realizados sobre essa temática, onde a baixa escolaridade foi evidenciada como um fator preditivo (Ferreira CO, et al., 2019, BRITO NM, et al., 2016).

Apesar de parte dos idosos (19\%) relatarem episódios anteriores de ISTs, não houve associação que justificasse a relação dessa variável com a prevalência das ISTs analisadas. Dentre as ISTs mais citadas em ordem decrescente tem-se: gonorreia, infecção por HPV, sífilis e herpes. Ressalta-se ainda que parte dos participantes não se sentiu seguro em responder se teve ou não uma infecção sexual por não saber exatamente o conceito de IST, o que poderia tornar esse dado mais significante ou não. Esse fato é de importante relevância pois assemelha-se aos resultados de Silva MP (2014), onde a maioria dos idosos afirmou não possuir conhecimento sobre as ISTs. Vale ressaltar que o Brasil não possui dados epidemiológicos acerca da maioria das ISTs, pois as mesmas não são de notificação compulsória, tornando difícil a real dimensão do número de casos de IST nessa população.

A maior parte dos idosos deste estudo $(77 \%)$ nunca havia sido testada para pesquisa de ISTs. Aqueles que tinham realizado exames previamente relataram ter sido durante check up de rotina. Durante a abordagem dos pacientes, alguns recusaram-se a participar da pesquisa e os principais motivos alegados para a recusa foram o medo em saber que são portadores de alguma nova enfermidade, além das que já possuíam; o fato de associar essas doenças ao homossexualismo, acreditando não serem portadores por 
serem heterossexuais; o fato de relacionarem a doença com estado civil, já que algumas mulheres referiram acreditar estar imunes pelo fato de relacionar-se apenas com seus maridos e a associação das doenças com prostituição.

Devido às justificativas supracitadas, é possível inferir como o conhecimento acerca destas doenças ainda é cercado de desinformações e crenças que precisam ser modificados através de informação e educação sobre esses assuntos.

\section{CONCLUSÃO}

No tocante a amostra analisada, observou-se uma alta prevalência para as IST na terceira idade, em especial para sífilis, a qual apresentou um número expressivo de casos. Evidenciou-se a associação entre ter IST e ser homem, ter um parceiro sexual fixo e não utilizar o preservativo rotineiramente. Os resultados apontam uma vulnerabilidade, no que tange a essa faixa etária da população, em vários aspectos biopsicossociais, pois desmitifica paradigmas da velhice assexuada para um envelhecimento ativo em todos os âmbitos. Espera-se que este estudo possa subsidiar cientificamente as tomadas de decisão, estimulando a adoção de estratégias mais efetivas de combate à IST na terceira idade.

\section{AGRADECIMENTOS}

Agradeçemos aos profissionais do Centro de Testagem e Aconselhamento (CTA) e à Secretaria Municipal de Saúde que auxiliaram na realização deste estudo.

\section{REFERÊNCIAS}

1. AFFELDT AB, et al. Perfil de pessoas idosas vivendo com HIV/aids em Pelotas, sul do Brasil, 1998 a 2013. Epidemiologia e Serviços de Saúde, 2015;24(1):79-86.

2. AGUIAR RB, et al. Idosos vivendo com HIV - comportamento e conhecimento sobre sexualidade: revisão integrativa. Ciência \& Saúde Coletiva, 2020;25(2):575-584.

3. ALENCAR RA. O idoso vivendo com HIV/AIDS: a sexualidade, as vulnerabilidades e os enfrentamentos na atenção básica. Tese (Doutorado em Enfermagem) - Escola de Enfermagem. Universidade de São Paulo, São Paulo, 2012;163p.

4. ANDRADE J, et al. Vulnerabilidade de idosos a infecções sexualmente transmissíveis. Acta Paulista de Enfermagem, 2017;(30)1:8-15.

5. ARAÚJO CAD. Ser idoso, sexualidade e cuidados preventivos no atual cenário da maior longevidade e envelhecimento populacional: Estudo de caso no município de Natal/RN. TESE (Pós-graduação em demografia) Universidade Federal de Minas Gerais, Minas Gerais, 2018; 127p.

6. BASTOS LM, et al. Avaliação do nível de conhecimento em relação à Aids e sífilis por idosos do interior cearense, Brasil. Ciência \& Saúde Coletiva, 2018; 23(8): 2495-2502.

7. BRASIL. Boletim epidemiológico de Sífilis. Sistema de vigilância em Saúde. Brasília: MS, 2019a.

8. BRASIL. Boletim HIV/Aids. Sistema de vigilância em Saúde. Brasília: MS, 2019b.

9. BRASIL. Hepatites Virais. Sistema de vigilância em Saúde. Brasília: MS, 2019c.

10. BRITO NM, et al. Idosos, infecções sexualmente transmissíveis e aids: conhecimentos e percepção de risco. ABCS Health Sciences, 2016; 41(3): 140-145.

11. CERQUEIRA MB, et al. Idosos de Montes Claros (MG) e HIV/Aids: conhecimentos e percepções. Revista Unimontes Científica, 2016; 18(1): 1-8.

12. DORNELAS JN, et al. Doenças sexualmente transmissíveis em idosos: uma revisão sistemática. Ciência e Saúde coletiva, 2015; 20(12): 3853-3864.

13. FERREIRA CD, et al. Vulnerabilidade a infecções sexualmente transmissíveis em idosos usuários de um centro de testagem e aconselhamento. Arquivos de Ciências da Saúde da UNIPAR, 2019; 23(3): 1-9.

14. GNOATTO FR, et al. Conhecimento de idosos participantes de centros de convivência acerca do hiv/aids. Research, Society and Development, 2020; 9(4): 1-10.

15. INSTITUTO BRASILEIRO DE GEOGRAFIA E ESTATíSTICA (IBGE). Censo Demográfico. Disponível em: https://cidades.ibge.gov.br/brasil/rr/boa-vista/panorama. Acesso em: 11 jun. 2020

16. LAROQUE MF, et al. Sexualidade do idoso: comportamento para a prevenção de DST/AIDS. Revista Gaúcha de Enfermagem, 2011; 32(4): 774-780. 
17. MIRANDA GM, et al. O envelhecimento populational brasileiro: desafios e consequências sociais atuais e futuras. Revista Brasileira de Geriatria e Gerontologia, 2016; 19(3): 507-519.

18. MOURA DS, et al. Sexuality in the elderly: a discussion about the measures of prevention of hiv/aids. Revista Ciencia \& Saberes, 2017; 3(1):407-415.

19. MOURA MMS, et al. Vulnerability to acquired immune deficiency syndrome in human perception of the elderly. Revista de Enfermagem da UFPI, 2014; 3(1): 100-106.

20. PASCUAL CP. A Sexualidade do idoso vista com novo olhar. São Paulo: Edições Loyola, 2002.

21. SILVA BN, et al. Panorama epidemiológico da Aids em idosos. Hygeia - Revista Brasileira de Geografia Médica e da Saúde, 2018; 14(29): 80-88.

22. SILVA JDB. et al. Vulnerabilidade às infecções sexualmente transmissíveis/ aids em idosos, 2017; 53(1): 19-24.

23. SILVA MP. Aspectos socioepidemiológicos das infecções sexualmente transmissíveis em idosos. Dissertação (Pósgraduação em Enfermagem) - Universidade Federal do Pará, Pará, 2014; 64p

24. SUZMAN R, et al. Health in an ageing world—what do we know? The Lancet, 2015; 385(9967): 484-486.

25. TERRA NL. Sexualidade, menopausa, andropausa, e disfunção erétil no envelhecimento: Compreensão e manejo. Porto Alegre: EDPUCRS, 2014.

26. THEIS LC, GOUVÊA DL. Percepção dos idosos em relação a vida sexual e as infecções sexualmente transmissíveis na terceira idade. Revista Brasileira de Ciências da Saúde, 2019; 23(2): 197-204.

27. VASCONCELOS AM, GOMES MM. Transição demográfica: a experiência brasileira. Epidemiologia e Serviços de Saúde, 2012; 21(4): 539-548.

28. VIEIRA KFL, et al. A Sexualidade na Velhice: representações sociais de idosos frequentadores de um grupo de convivência. Psicologia: Ciência e Profissão, 2016; 36(1): 196-209. 